Research article

\title{
Prevalence and Antimicrobial Susceptibility Pattern of Pseudomonas aeruginosa Isolated from Various Clinical Samples in a Tertiary Care Hospital
}

\author{
Surya Narayan Mahaseth', Lokeshwar Chaurasia' ${ }^{2}$, Brajesh Jha ${ }^{3}$, Raj Kumari Sanjana ${ }^{3}$ \\ Department of Microbiology \\ Janaki Medical College, Janakpur, Nepal \\ ${ }^{1}$ Assistant professor, Department of Microbiology, Janaki Medical College, Janakpur, Nepal \\ 2 Assistant professor, Department of Pharmacology, Janaki Medical College, Janakpur, Nepal \\ ${ }^{3}$ Associate professor, Department of Microbiology, College of Medical Sciences, Bharatpur, Chitwan, Nepal
}

\begin{abstract}
Background and Objectives: Pseudomonas aeruginosa is one of the leading causes of hospitalacquired infections. Increased resistance in this organism continues to pose a significant threat to patient care because of limited therapeutic options. The main objective of this study was to find out the prevalence and current antimicrobial susceptibility pattern of $P$. aeruginosa isolates obtained from various clinical samples at a tertiary care hospital.

Material and Methods: The study was conducted in a tertiary care hospital in Bharatpur, Chitwan, Nepal on 453 isolates of Pseudomonas aeruginosa from various clinical samples. The colonies which were grown on culture media were identified by different standard biochemical tests. Antimicrobial susceptibility testing was done using Kirby-Bauer disc diffusion method and the results were interpreted according to the CLSI guidelines. Quality control of the test was done by standards ATCC strain of $P$. aeruginosa 27853.
\end{abstract}

Results: This present study revealed the prevalence rate of $P$. aeruginosa was $11.29 \%$. Piperacillintazobactam was the most sensitive chemotherapeutic agent with $94.26 \%$ susceptibility rate, followed by imipenem $89.40 \%$ and levofloxacin $88.08 \%$. Amikacin showed better susceptibility rate $67.33 \%$ than that of gentamicin $48.78 \%$; the susceptibility rate to cephalosporin and aztreonam was relative very low. Most of the P. aeruginosa strains were isolated from clinical samples like sputum 206, urine 81, respiratory secretion 76, and pus 35 . Out of 453 clinical isolates, 167(36.86\%) clinical isolates of $P$. aeruginosa were found to be MDR.

Conclusion: Most of the P. aeruginosa strains were isolates from sputum, urine, respiratory secretions and pus samples and were found to be MDR. Piperacillin-tazobactam was the most sensitive chemotherapeutic agent followed by Imipenem, levofloxacin.

Key words: Pseudomonas aeruginosa, Antibiotic Susceptibility Testing, Antipseudomonal Agents, Tazobactam, Aztreonam, Multi Drug Resistance (MDR) 
opportunistic bacterium, which is responsible for one of the leading causes of nosocomial and community acquired infections [1]. It is also responsible for a variety of systemic infections like urinary tract infections, respiratory tract infections, gastrointestinal tract infections, dermatitis, bacteremia, soft tissue infections, bone and joint infections [2]. It is one of the important bacterial pathogens isolated from various samples. Despite advances in medical and surgical care and introduction of wide variety of antimicrobial agents having anti-pseudomonal activities, life-threatening infections caused by $P$. aeruginosa continues to cause complications in nosocomial infections [3].

Pseudomonas aeruginosa has naturally resistant to many antibiotics due to the permeability barrier afforded by its outer membrane lipopolysaccharide (LPS). There are variety of mechanisms involved in the resistance of $P$. aeruginosa such as efflux pumps [4], production of ESBLs (extended spectrum beta-lactamases) [5], MDR, target site or outer membrane modification [3], biofilm formation and aminoglycoside modifying enzymes [6]. ESBLs are an important reason of bacterial resistance throughout the world [5]. Microbiologists, clinicians, infection control professionals and scientists engaged in finding new antimicrobial agents are now facing distinctive challenge to ESBL producing isolates.

In addition to its intrinsic resistance to various antibiotics, it also readily acquires resistance to the potentially active agents [7]. Since some of the resistance markers are carried by plasmids, the threat to human health is compounded by the possibility of transmission of markers to other Gramnegative pathogens [8]. Resistance to anti- pseudomonal antibiotics is increasing worldwide. This situation has been compounded by the lack of new classes of anti-pseudomonal drugs [9]. Therefore, knowledge of current drug resistance pattern of the common pathogenic bacteria in a particular region is useful in clinical practice. Hence, the present study was conducted to find out prevalence and the antimicrobial susceptibility pattern of $P$. aeruginosa isolates obtained from various clinical samples at Microbiology department in a tertiary care hospital, Bharatpur, Chitwan, Nepal.

\section{MATERIAL AND METHODS}

The study was conducted at Department of Microbiology, Janaki Medical College Teaching Hospital, Janakpurdham, Nepal with approval from the Institutional Review/Ethical Committee. All the clinical samples including sputum, pus, urine, respiratory samples, tissue and blood received in the Microbiology laboratory for culture and sensitivity were processed. After initial Gram staining, they were inoculated on blood agar and MacConkey's agar. The inoculated culture plates were incubated aerobically at $37^{\circ} \mathrm{C}$ for 24 hours [10].

Identification: Following the appearance of bacterial growth, only isolates of Pseudomonas aeruginosa were included in this study. The standard biochemical tests were applied for identification.

Details of the patient: Demographic details including age, sex, ward/critical unit were noted.

Antibiotic susceptibility test: Antimicrobial susceptibility testing was performed on Mueller-Hinton agar (MHA) plates by KirbyBauer disc diffusion method, according to Clinical Laboratory Standards Institute (CLSI) 
guidelines. All Pseudomonas aeruginosa isolates were subjected to antimicrobial susceptibility test as per CLSI guidelines [11].

Following antibiotic discs from HI- MEDIA were used for antibiotic susceptibility test: piperacillin $(100 \mu \mathrm{g}), \quad$ piperacillin + tazobactum $(100 / 10 \mu \mathrm{g})$, ticarcillin + tazobactum $(75 / 10 \mu \mathrm{g})$, ceftazidime $(30 \mu \mathrm{g})$, cefotaxime $(10 \mu \mathrm{g})$, imipenem $(10 \mu \mathrm{g})$, meropenem $(10 \mu \mathrm{g})$, amikacin $(30 \mu \mathrm{g})$, gentamicin $(10 \mu \mathrm{g})$, ciprofloxacin $(5 \mu \mathrm{g})$, levofloxacin $(5 \mu \mathrm{g})$ and aztreonam $(50 \mu \mathrm{g})$.

Pseudomonas aeruginosa, ATCC 27853 strains, was used for quality control.

\section{RESULTS}

The present study was carried out in the Microbiology laboratory, College of Medical Sciences and Teaching Hospital (COMS-TH), Bharatpur, Chitwan, Nepal for 1 year. A total of 9139 samples were received for culture and sensitivity in Bacteriology Diagnostic Laboratory, of which 4013 samples showed growth on culture. Among 4013 cultured organism, 453 isolates were identified as $P$. aeruginosa (11.29\%). The samples from which we cultured were sputum, urine, pus, wound swab, blood, respiratory secretions and others. Out of 439 isolated P. aeruginosa, the most common samples given positive growth were sputum 206 (45.47\%), urine 81 (17.88\%), respiratory secretions 76 (16.78\%), pus 35 (7.73\%), wound swab 21 (4.63\%), blood 14 (3.09\%) and other samples $20(4.41 \%)$ as shown in Table 1.

Among 453 cases of $P$. aeruginosa, $282(62.25 \%)$ cases were isolated from male patients and 171(37.75\%) were from female patients as shown in table 2 .
Table 1: Distribution of total isolates of $P$. aeruginosa strains from different samples

\begin{tabular}{|l|c|c|}
\hline \multicolumn{1}{|c|}{ Clinical samples } & Cases & $\begin{array}{c}\text { Percentage } \\
\text { (\%) }\end{array}$ \\
\hline Sputum & 206 & 45.47 \\
\hline Urine & 81 & 17.88 \\
\hline Pus & 35 & 7.73 \\
\hline Wound swab & 21 & 4.63 \\
\hline $\begin{array}{l}\text { Respiratory secretions (E.T. } \\
\text { tip/ bronchial wash etc) }\end{array}$ & 76 & 16.78 \\
\hline Blood & 14 & 3.09 \\
\hline $\begin{array}{l}\text { Others (CSF/semen/foley's } \\
\text { tip) }\end{array}$ & 20 & 4.41 \\
\hline Total & $\mathbf{4 5 3}$ & $\mathbf{1 0 0}$ \\
\hline
\end{tabular}

Table 2: Sex-wise distribution of $P$. aeruginosa strains

\begin{tabular}{|l|c|c|}
\hline Gender & $\begin{array}{c}\text { Number of } \\
\text { samples }\end{array}$ & Percentage (\%) \\
\hline Male & 282 & 62.25 \\
\hline Female & 171 & 37.75 \\
\hline Total & $\mathbf{4 5 3}$ & $\mathbf{1 0 0}$ \\
\hline
\end{tabular}

Table 3 shows majority of the isolates $(81.46 \%)$ were recovered from patients attending inpatient departments than from outpatient departments (18.54\%). Maximum number of $P$. aeruginosa was recovered from patients who were more than 40 upto 60 years of age (33.99\%) followed by those who were more than 60 years of age $(24.94 \%)$ as shown in table 4.

Table 3: Distribution of $P$. aeruginosa strains among outpatient department (OPD) and inpatient department (IPD)

\begin{tabular}{|l|c|c|}
\hline Department & $\begin{array}{c}\text { Number of } \\
\text { isolates }\end{array}$ & Percentage (\%) \\
\hline IPD & 369 & 81.46 \\
\hline OPD & 84 & 18.54 \\
\hline Total & $\mathbf{4 5 3}$ & $\mathbf{1 0 0}$ \\
\hline
\end{tabular}

Table 4: Age-wise distribution of isolates of $P$. aeruginosa

\begin{tabular}{|l|c|c|}
\hline Age in years & Total samples & Percentage (\%) \\
\hline $0-20$ & 75 & 16.56 \\
\hline $21-40$ & 111 & 24.50 \\
\hline $41-60$ & 154 & 33.99 \\
\hline$>60$ & 113 & 24.94 \\
\hline Total & $\mathbf{4 5 3}$ & $\mathbf{1 0 0}$ \\
\hline
\end{tabular}


Table 5 shows antibiotic sensitivity patterns of $P$. aeruginosa. We found that the most sensitive drug was piperacillin-tazobactam $94.26 \%$ (427) followed by imipenem $89.40 \%$ (405), levofloxacin 88.08\% (399), ticarcillin + tazobactam 87.86\% (398), meropenem 83.0\% (376) and piperacillin 74.83\% (339). In our study we also found $P$. aeruginosa MDR Strains from clinical isolates as shown in table 6.

Table 5: Antimicrobial susceptibility of $P$. aeruginosa isolates $(n=453)$ to various antibiotics

\begin{tabular}{|l|l|c|c|c|c|}
\hline S.No & Name of antibiotics & $\begin{array}{c}\text { Sensitivity } \\
\text { number of } \\
\text { samples }\end{array}$ & Sensitivity \% & $\begin{array}{c}\text { Resistant } \\
\text { number of } \\
\text { samples }\end{array}$ & Resistance \% \\
\hline 1. & Piperacillin & 339 & 74.83 & 114 & 25.16 \\
\hline 2. & Piperacillin+ tazobactum & 427 & 94.26 & 26 & 5.74 \\
\hline 3. & Ticarcillin+ tazobactum & 398 & 87.86 & 55 & 12.14 \\
\hline 4. & Meropenem & 376 & 83.00 & 77 & 16.99 \\
\hline 5. & Imipenem & 405 & 89.40 & 48 & 10.60 \\
\hline 6. & Gentamicin & 221 & 48.78 & 232 & 51.21 \\
\hline 7. & Amikacin & 305 & 67.33 & 148 & 32.67 \\
\hline 8. & Ciprofloxacin & 230 & 50.77 & 223 & 49.23 \\
\hline 9. & Levofloxacin & 399 & 88.08 & 54 & 11.92 \\
\hline 10. & Aztreonam & 282 & 62.25 & 171 & 37.75 \\
\hline 11. & Ceftazidime & 232 & 51.21 & 221 & 48.78 \\
\hline 12. & Cefotaxime & 241 & 53.0 & 212 & 46.80 \\
\hline
\end{tabular}

Table 6: Distribution of MDR P. aeruginosa isolates among clinical specimens

\begin{tabular}{|l|l|c|c|c|}
\hline S.No. & Clinical samples & Total number of samples & $\begin{array}{c}\text { Number of MDR } \\
\text { strains }\end{array}$ & $\begin{array}{c}\text { Percentage (\%) of } \\
\text { MDR isolation }\end{array}$ \\
\hline 1. & Sputum & 206 & 85 & 41.26 \\
\hline 2. & Urine & 81 & 21 & 25.92 \\
\hline 3. & Pus & 35 & 13 & 37.14 \\
\hline 4. & Wound swab & 21 & 12 & 57.14 \\
\hline 5. & Respiratory secretions & 76 & 28 & 36.84 \\
\hline 6. & Blood & 14 & 03 & 21.43 \\
\hline 7. & Others & 20 & 05 & 25.00 \\
\hline & Total & 453 & 167 & 36.86 \\
\hline
\end{tabular}

\section{DISCUSSION}

In this study, a total of 453 strains of $P$. aeruginosa were isolated and identified from various clinical samples from the outpatient and inpatients and their antimicrobial susceptibility patterns were determined. This present study revealed the prevalence rate of $P$. aeruginosa was $11.29 \%$ which is almost comparable to the prevalence rate shown in studies done in India 9.28\% [12], 13\% [13]. A similar study done in Kathmandu [14], Nepal in 2013 showed $17.05 \% \quad P$. aeruginosa isolates. A higher prevalence rate $(32-40 \%)$ was also documented by other studies [15, 16].

In our study, we found that most of the cases belonged to older age group of 41-60 years (33.99\%) followed by elderly age group of $>60$ years $(24.94 \%)$. This could be explained 
patients $62.25 \%$ constituted a larger group in our study. Other studies from India, Nepal have also shown similar findings [17, 18]. The distribution of specimen of $P$. aeruginosa may vary with each hospital as each hospital has a different environment and facility associated within. The most common source of the isolate in our study was sputum (45.47\%) followed by urine $(17.88 \%)$ and only $7.73 \%$ of the isolates were recovered from pus. Shresthaet al. also reported maximum percentage of $P$. aeruginosa from sputum, urine (36.27\% each) and pus (9.8\%) [18]. Other studies reported maximum isolation from pus followed by urine and sputum [19].

We studied resistance pattern of $P$. aeruginosa against 12 antimicrobial agents; $P$. aeruginosa isolates were found most sensitive to piperacillin + tazobactam (94.26\%) followed by imipenem (89.40\%) and this may be due to the restricted use of piperacillin + tazobactam and imipenem in this hospital. Imipenem is a carbapenem antibiotic which is highly active against $P$. aeruginosa. Our study showed only $10.60 \%$ of resistance to imipenem which was almost similar to the resistance rate revealed by study done in a different university of Kathmandu (9.1\% and $15.4 \%)[20,21]$. Higher rate of resistance was seen in study done by Taneja et al. (36.4\%) [22] and Chaudhary et al. (66.5\%) [23].

In our study, levofloxacin $11.92 \%$ resistant followed by ciprofloxacin $49.23 \%$ resistant, while meropenem only $16.99 \%$ resistant and amikacin $32.67 \%$ resistant were detected to be the most effective drugs for routine use against the $P$. aeruginosa strains investigated. Among the quinolones and aminoglycosides, our study detected $49.23 \%$ resistance to ciprofloxacin which is comparable to the study done by Yadav et al. who reported 49\% resistance to ciprofloxacin [13]; similarly higher rate of resistance had also been reported from Kerala, India (40.5\%) [24], and from Malaysia (92\%) [25]. An earlier study reported from Kathmandu, Nepal showed amikacin was $81.4 \%$ sensitive [26], In this study, we got amikacin $67.33 \%$ sensitive among $P$. aeruginosa. In other study, high percentage of resistance to aminoglycosides had been reported from India [27], Bangladesh [28]. In our study, piperacillin + tazobactam showed $94.26 \%$ sensitive whereas piperacillin alone showed $74.83 \%$ sensitive followed by ticarcillin + tazobactam $87.86 \%$ sensitive. In other study, piperacillin alone tested showed a resistance rate of 25.16\% reported from Chhattisgarh, India [13]. Therefore, it indicates beta-lactamase inhibitor markedly expands the spectrum of activity of drug. Thus, it determines that the combination drug should be the preferred choice against $P$. aeruginosa.

We found that $P$. aeruginosa was $48.78 \%$ resistant to ceftazidime, third-generation cephalosporin drug and $46.80 \%$ resistant to cefotaxime. Much higher resistance to thirdgeneration cephalosporin 95\%, 75\%, and $86 \%$, had been reported in studies done in Nepal [26], India [27] and Bangladesh [28].

We found sensitivity to levofloxacin was 88.08\%. Thus, levofloxacin should be included in the treatment regimen for the $P$. aeruginosa. Another significant finding in this study was the rate of multidrug resistance (MDR), which was found to be $36.86 \%$; similarly, MDR rate of $19.6 \%$ from Malaysia [29] and 20.69\% from Kathmandu, Nepal [21] had been reported. A higher rate of MDR had been reported from studies conducted from Nepal (89.4\%) [26] and 100\% MDR $P$. aeruginosa isolates from Iran [30]. This study has a few limitations that are molecular 
typing, plasmid profile and study of mechanism of development of MDR strain, which would provide much-needed detail information and there should also be analysis of ESBL-producing $P$. aeruginosa which is a major cause of nosocomial infection with MDR strains in hospital [31, 32].

\section{CONCLUSION}

The prevalence and sensitivity of $P$. aeruginosa often varies between communities, hospitals in the same community and among different patient populations in the same hospital. In our study, we found results that clearly indicate the evolution of MDR stains and the occurrence of resistance to various antipseudomonal agents among the P. aeruginosa isolates. We suggest a more restricted and more rational use of drugs in the treatment of $P$. aeruginosa infection in hospital setting. Increase in antibacterial resistance in $P$. aeruginosa is a cause of concern. Regular antimicrobial susceptibility monitoring is essential for local, regional and national level isolates. This would help prescribing the right combination of chemotherapeutic agent and prevent the emergence of MDR strains of $P$. aeruginosa.

\section{ACKNOWLEDGEMENTS}

We thank Mr. Ganesh Kumar Singh (technical staff) of Microbiology Laboratory of COMS-TH for his help in performing the various laboratory investigations.

\section{Source of Funding: none}

\section{Conflict of interest: none}

\section{REFERENCES}

1. Lambert PA. Mechanisms of antibiotic resistance in Pseudomonas aeruginosa. J R Soc Med. 2002;95 Suppl41:22-62.
2. Prakash HR, Belodu Rashmi, Karangate N, Sonth S, Anitha MR, Vijayanath V. Antimicrobial susceptibility pattern of Pseudomonas aeruginosa strains isolated from clinical sources. Journal of Pharmaceutical and Biomedical Sciences (JPBMS). Vol. 14, Issue 14.

3. Rajat RM, Ninama GL, Mistry K, Parmar R, Patel K, Vegad MM. Antibiotic Resistance Pattern In Pseudomonas aeruginosa Species Isolated At A Tertiary Care Hospital, Ahmadabad. National Journal of Medical Research, 2012 Vol 2 Issue 2 April-June.

4. Li XZ, Nikaido H, Poole K. Role of mexA-mexB oprM in antibiotic efflux in Pseudomonas aeruginosa. Antimicrob Agents Chemother. 1995; 39: 1948-53.

5. Manchanda V, Singh NP. Occurrence and detection of Amp C betalactamases among Gram negative clinical isolates using a modified three dimensional test at Guru Tegh Bahadur Hospital, Delhi, India. J Antimicrob Chemother. 2003; 51: 415-18.

6. Mahmoud AB, Zahran WA, Flindawi GR, Lebib A, Galal R. Prevalence of multidrug - resistant Pseudomonas aeruginosa in patients with nosocomial infection at a university hospital in Egypt, with special reference to typing methods. J Virol Microbiol. 2013; 2013:153.

7. Forbes BA, Sahm DF, Weissfeld AS. Bailey and Scott's Diagnostic Microbiology. 11th ed. St. Louis. Mosby Inc.; 2002. p. 389-94.

8. Balows A, Duerden BI. Topley \& Wilson's Systemic Bacteriology. 9th ed., Vol. 2. London: Arnold; 1998.

9. Fauci AS, Branuwald E, Kasper DL, Hauser SL, Longo DL, Jameson JL, et al. editors. Harrisons Principles of Internal Medicine. 17th ed., Vol. 1. New York, NY: McGraw-Hill; 2008. p. 954.

10. Ian Phillip. Identification of Pseudomonas Aeruginosa In The Clinical Laboratory. Med.Microbiol.-Vol.2 (1969)

11. Clinical Laboratory Standard Institute (2010) Performance Standards for Antimicrobial Susceptibility Testing: Twentieth Informational Supplement, M100S20. Clinical and Laboratory Standards Institute.

12. Srinivas B, Lalitha Devi D, Narasinga Rao B. A prospective study of Pseudomonas aeruginosa and its antibiogram in a Teaching Hospital of Rural setup. J Pharm Biomed Sci. 2012; 22 (18): 1-5.

13. Yadav VC, Vepada RK, Jaiswal MK, Singh K. A study of antibiotic sensitivity pattern of 
Pseudomonas aeruginosa isolated from a tertiary care hospital in South Chhattisgarh. International Journal of Medical Science and Public Health 2017; 6 (3): 600-605.

14. Chandel A, Raza Mohammad S. Antimicrobial susceptibility patterns of Pseudomonas aeruginosa clinical isolates at a tertiary care hospital in Kathmandu, Nepal. Asian J Pharm Clin Res. 2013; 6 (3): 235-58.

15. Tahira M, Mohammed AM, Galnaz K, Mustafa K. Pseudomonas aeruginosa in chronic suppurative otitis media: sensitivity spectrum against various antibiotics in Karachi. J Ayub Med Coll Abbottabad. 2009;21 (2): 120-23.

16. Anupurba S, Bhattacharjee A, Garg A, Sen MR. Antimicrobial susceptibility of Pseudomonas aeruginosa isolated from wound infections. Indian J Dermatol 2006; 51: 286-8.

17. Rakesh MR, Govind LN, Kalpesh M, Rosy P, Kavu P, Vegad MM. Antibiotic resistance pattern in Pseudomonas aeruginosa species isolated at a tertiary care hospital, Ahmadabad. Natl J Med Res. 2012;2(2):156-9.

18. Shrestha S, Amatya R, Adhikari RP. Prevalence and antibiogram of Pseudomonas aeruginosa isolated from clinical specimens in a Teaching Hospital, Kathmandu. Nepal Med Coll J 2015; 17 (3-4): 132-135.

19. Angadi $K$ M, Kadam $M$, Modak $M \quad S$, Bhatavdekar S M, Dalal B A, Jadhavvar S R et al. Detection of antibiotic resistance in Pseudomonas aeruginosa isolate with special reference to metallo-beta-lactamases from a tertiary care hospital in Western India. International J Microbio Res. 2012; 4 (7): 2958.

20. Shrestha RK, Dahal RK, Mishra SK, Parajuli K, Rijal BP, Sherchand JB et al. Ventilator Associated Pneumonia in Tertiary Care Hospital, Maharajgunj, Kathmandu, Nepal. Journal of Institute of Medicine. 2013; 35 (3): 21-28.

21. Mishra SK, Acharya J, Kattel HP, Koirala J, Rijal BP, Pokhrel BM. Metallo-beta-lactamase producing gram negative bacterial isolates. J Nepal Health Res Counc 2012 Sep; 10 (22): 208-13.

22. Taneja N, Maharwal S, Sharma M. Imipenem resistance in Non fermenters causing nosocomial urinary tract infections. Ind J Med Sci. 2003; 77 (7): 294-299.

23. Chaudhary M, Payasi A. Rising Antimicrobial Resistance of Pseudomonas aeruginosa
Isolated from clinical specimens in India. J Proteomics Bioinform 2013; 6 (1): 5-9.

24. Ahmed SM, Jakribettu RP, Kottakutty S, Arya B, Shakir VP. An emerging multi-drug resistant pathogen in a tertiary care center in North Kerala. Ann Biol Res. 2012;3(6):2794-9.

25. Fazlul MK, Zaini MZ, Rashid MA, Nazmul MH. Antibiotic susceptibility profiles of clinical isolates of Pseudomonas aeruginosa from Selayang Hospital, Malaysia. Biomed Res. 2011;22(3):263-66.

26. Bhandari S, Banjara MR, Lekhak B, Bhatta DR, Regmi SR. Multi-drug and pan-drug resistant Pseudomonas aeruginosa: A challenge in postantibiotic era. Nepal J Sci Tech. 2012;13(2):197-202.

27. Arora D, Jindal N, Kumar R, Romit. Emerging antibiotic resistance in Pseudomonas aeruginosa. Int J Pharm Pharm Sci. 2011;3(2):82-4.

28. Rashid A, Chowdhury A, Rahman SH, Begum SA, Muazzam N. Infections by Pseudomonas aeruginosa and antibiotic resistance pattern of the isolates from Dhaka Medical College Hospital. Banglad J Med Microbiol. 2007;1(2):48-51.

29. Pathmanathan SG, Samat NA, Mohamed R. Antimicrobial susceptibility of clinical isolates of Pseudomonas aeruginosa from a Malaysian Hospital. Malays J Med Sci. 2009;16(2):27-32.

30. Moazami-Goudarzi S, Eftekhar F. Assessment of carbapenem susceptibility and multidrugresistance in Pseudomonas aeruginosa burn isolates in Tehran. Jundishapur J Microbiol. 2013;6(2):162-5.

31. Nwankwo EO, Shuaibo SA. Antibiotic susceptibility pattern of clinical isolates of Pseudomonas aeruginosa in a tertiary health institution in Kano, Nigeria. J Med Biomed Sci. 2010;17:37-40.

32. Anjum F, Mir A. Susceptibility pattern of Pseudomonas aeruginosa against various antibiotics. Afr J Microbiol Res. 2010;4 (10):1005-12.

\section{Correspondence to:}

Dr Surya Narayan Mahaseth

Assistant professor

Department of Microbiology

Janaki Medical College

Janakpur, Nepal.

Email ID: dosurya@gmail.com

Contact number: +977-9851012818 